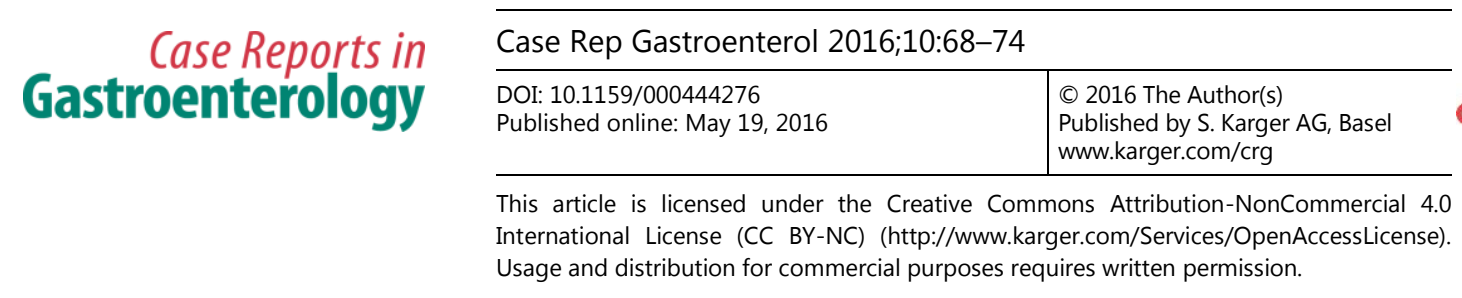

\title{
A Delayed Diagnosis of Chronic Mesenteric Ischaemia: The Role of Clinicians" Cognitive Errors
}

\author{
Sern Wei Yeoh \\ Department of Gastroenterology and Hepatology, Eastern Health, \\ Melbourne, Vic., Australia
}

\section{Keywords}

Mesenteric vascular insufficiency - Weight loss - Diarrhea - Nausea - Delayed diagnosis .

Diagnostic errors · Angioplasty

\begin{abstract}
Chronic diarrhoeal illnesses with nausea and weight loss are a common indication for gastroenterology review. While many such cases have intra-luminal aetiologies, such as inflammatory bowel disease, coeliac disease or other malabsorptive conditions, with many other cases due to functional gut disorders or systemic malignancy, clinicians must also keep vascular disorders in mind. Here we report a patient with a delayed diagnosis of chronic mesenteric ischaemia after 6 months of gastrointestinal symptoms strongly mimicking an alternative diagnosis such as inflammatory bowel disease due an atypical predominance of nausea and diarrhoea rather than pain. We briefly review the literature on treatment of this condition but also discuss with particular attention the sequence of cognitive errors made by clinicians that led to a diagnostic delay, inviting readers to thus reflect on how such errors can be minimised in their practice.

(C) 2016 The Author(s)

Published by S. Karger AG, Basel
\end{abstract}




\section{Case Reports in Gastroenterology}

Case Rep Gastroenterol 2016;10:68-74

(c) 2016 The Author(s). Published by S. Karger AG, Basel www.karger.com/crg

Yeoh: A Delayed Diagnosis of Chronic Mesenteric Ischaemia: The Role of Clinicians' Cognitive Errors

\section{Introduction}

Atherosclerotic stenoses of the coeliac, superior mesenteric and inferior mesenteric arteries are common. In a retrospective review of 980 angiographic studies by Thomas et al. [1], $8 \%$ had significant stenosis of at least one mesenteric artery. However, extensive collateral vessels often form between the territories of these major arteries, such that compensatory blood supply is achieved in the setting of single vessel stenosis. Most cases of symptomatic chronic mesenteric ischaemia therefore occur in the setting of 2 or 3 mesenteric arteries being affected [2]. Mesenteric blood flow normally increases from $25 \%$ of total cardiac output to $35 \%$ or more after a meal, but in the absence of sufficient perfusion, ischaemia ensues with anaerobic glycolysis and lactate production by intestinal enterocytes. Cell death occurs due to disruption of membrane pumps, leading to reduced epithelial barrier function and translocation of intestinal bacteria into the bloodstream [3]. This initiates a local inflammatory response that in turn leads to the classic symptoms of post-prandial pain and nausea with subsequent anorexia, avoidance of meals and weight loss [2]. Chronic mesenteric ischaemia is a rare condition, however, with only 13 of 980 patients in the study by Thomas et al. [1] having significant symptomatic multi-vessel stenosis. Therefore, it may be overlooked by gastroenterologists in favour of more common luminal or functional differential diagnoses. We present a case where a diagnosis of chronic mesenteric ischaemia was delayed due to atypical symptomatology, leading to consecutive cognitive errors by the treating clinicians.

\section{Case Presentation}

A 67-year-old man presented to clinic with 4 months of increasing diarrhoea and weight loss from 74 to $60 \mathrm{~kg}$. He had a height of $1.91 \mathrm{~m}$ and a body mass index of 16.4. He opened his bowels up to 8 times a day with loose motions, though without associated blood or mucus, and had not improved with trials of gluten-free and lactose-free diets. Prior to this, he had never had gastrointestinal symptoms. His past history was significant for Klinefelter's syndrome requiring parenteral testosterone replacement, 2 previous lumbar laminectomies, and chronic obstructive pulmonary disease with a 30 pack-year history of cigarette smoking that had ceased 4 years prior.

Multiple outpatient investigations were performed, all with negative results. These included full blood count; renal function and liver function tests; serum levels of iron, vitamin B12, folate and albumin; erythrocyte sedimentation rate and C-reactive protein; thyroid function tests; serology for human immunodeficiency virus hepatitis B and hepatitis C; stool cultures and parasite screening, and anti-tissue transglutaminase antibodies. Computed tomography (CT) scans of his chest, abdomen and pelvis revealed only a 1-cm left adrenal adenoma which appeared benign, uncomplicated cholelithiasis and atheroma within the abdominal aorta. Gastroscopy and colonoscopy were macroscopically unremarkable, with biopsies of the stomach, duodenum, terminal ileum and colon showing no abnormalities.

On a second clinic review 2 months later, his symptoms had progressed to include nausea beginning $1 \mathrm{~h}$ after meals, persisting for several hours and causing secondary anorexia. His diarrhoea continued, and a further kilogram of weight had been lost. Further outpatient tests included a nuclear medicine gastric emptying study with a negative result; although faecal calprotectin via the Quantum Blue method was elevated at $145 \mu \mathrm{g} / \mathrm{g}$, indicating a high probability of inflammation in the gastrointestinal tract. 
Yeoh: A Delayed Diagnosis of Chronic Mesenteric Ischaemia: The Role of Clinicians Cognitive Errors

Three weeks later, prior to a planned clinic appointment, he was admitted to the hospital with ongoing nausea and diarrhoea which was impossible to be managed at home with anti-emetic and anti-diarrhoeal medications. New symptoms included cramping lower abdominal pain associated with the nausea, radiating to the back. Due to the elevated calprotectin, CT enteroclysis of the small bowel was performed to assess for Crohn's disease, with a negative result. Further blood tests ensued with negative results including lipase, lactate, anti-nuclear antibodies, rheumatoid factor, complement levels, IgG subclasses, vasoactive intestinal peptide and gastrin, cortisol and protein electrophoresis. Urine tests for electrophoresis, 24-hour catecholamines and 5-hydroxyindoleacetic acid were unremarkable, as were faecal elastase and CT of the brain.

His symptoms improved significantly during a 14-day hospital admission without any change to medications. He was highly anxious, and further history revealed recent major psychosocial stressors, as well as several episodes of left leg pain upon mobilising that the patient ascribed to referred pain from his previous laminectomies. He was therefore given a presumptive diagnosis of a diarrhoeal predominant irritable bowel syndrome with a possible contribution of neuropathic pain radiating from his back. Amitriptyline $12.5 \mathrm{mg}$ daily was started, as well as a diet low in fermentable oligosaccharides, disaccharides, monosaccharides and polyols (FODMAPs). His symptoms improved further over several days, and an outpatient psychiatric review was arranged. However, mild abdominal pain recurred on the evening prior to planned discharge and his abdomen was auscultated by junior medical staff, with a vascular bruit heard in the epigastrium. Mesenteric vessel ultrasound demonstrated $>70 \%$ stenoses in the coeliac and superior mesenteric (fig. 1) vessels, though as his symptoms abated again over the next $24 \mathrm{~h}$ the significance of these was initially unclear. The patient self-discharged, wishing to be further investigated as an outpatient.

He represented several days later with severe abdominal pain, elevated white cell count of $16 \times 10^{9} / \mathrm{l}$ and C-reactive protein of 52 , though with normal serum lactate and lipase. His arterial carboxyhaemoglobin was measured for the first time and was elevated at 5.2\%. Upon questioning, the patient admitted to having restarted smoking at home 6 months prior, corresponding to the onset of his symptoms, but abstaining whilst in hospital. Mesenteric CT angiography demonstrated short $90 \%$ ostial stenoses of the coeliac and superior mesenteric arteries (fig. 2) as well as an occluded left common iliac artery. The inferior mesenteric artery was patent.

His diagnosis was revised to one of chronic mesenteric ischaemia due to two-vessel atherosclerosis, with his left leg pain also being due to arterial insufficiency. Fluoroscopically guided balloon dilatation and insertion of 2 self-expanding stents (6-mm and 7-mm diameter) into the superior mesenteric artery over a guidewire was performed (fig. 3). It was intended that collateral vessels from the reperfused superior mesenteric artery would subsequently supplement blood flow to the territories of the stenosed coeliac artery. Immediate relief in abdominal symptoms ensued, and aspirin $100 \mathrm{mg}$ daily and clopidogrel $75 \mathrm{mg}$ daily were commenced to prevent stent thrombosis. At 1 month after discharge, he was still free from abdominal symptoms, had stopped smoking and gained $5 \mathrm{~kg}$ of weight. He is planned for open revascularisation of his left leg via femoral bypass in the future.

\section{Discussion}

This patient was subject to diagnostic delay for several reasons, exemplifying common cognitive errors described in clinical medicine. Firstly, his symptoms consisted primarily of 
Yeoh: A Delayed Diagnosis of Chronic Mesenteric Ischaemia: The Role of Clinicians Cognitive Errors

diarrhoea and nausea for the majority of his illness, with pain being a very late feature. This is unusual: in one retrospective review of 54 cases of mesenteric ischaemia, pain was present in $98 \%$ of patients, with diarrhoea only in $24.5 \%$ and nausea in $2 \%$ [4]. The clinicians therefore applied a 'representativeness heuristic', wherein the patient exhibited a cluster of symptoms or 'illness script' common to those with inflammatory bowel disease, rather than mesenteric ischaemia [5]. This bias was strengthened by an elevated faecal calprotectin. However, this is not specific for inflammatory bowel disease as calprotectin is raised in myriad intestinal conditions such as infections, malabsorptive syndromes and neoplasms [6].

Secondly, the patient's diagnostic work-up was coordinated by gastroenterologists, which led to two further cognitive errors being applied: the 'availability heuristic', whereby clinicians' regular experience with intra-luminal aetiologies such as inflammatory bowel disease, rather than vascular aetiologies, biased the initial investigations [7], and 'ascertainment bias', whereby clinicians investigated for conditions (such as inflammatory bowel disease) that they hoped to find due to their familiarity with subsequent treatment [8].

Thirdly, clinicians were falsely reassured by unremarkable previous abdominal imaging including 2 CT scans and ultrasound. However, none of these previous studies targeted mesenteric vessels. Also falsely reassuring was the absence of biochemical markers of malnutrition such as anaemia or hypoalbuminaemia; as well as frank lactataemia. However, serum lactate levels are rarely raised in chronic mesenteric ischaemia due to the capacity of the liver to metabolise lactate delivered via the portal vein [4]. As such, serum lactate is elevated only in cases of critical transmural ischaemia.

Fourthly, the patient's improvement during the hospital stay in the setting of psychosocial stressors engendered a diagnosis of a functional gut disorder and the dual cognitive errors of 'fundamental attribution error', whereby clinicians frustrated by an exhaustive course of investigation attributed symptoms to the patient's personal characteristics [9], as well as 'premature closure', whereby the search for further organic aetiologies was abandoned [10]. We retrospectively theorise that his symptoms improved in the hospital due to smoking cessation, leading to a lower blood carboxyhaemoglobin to oxyhaemoglobin ratio and thereby improving tissue perfusion.

The treatment of chronic mesenteric ischaemia centres around revascularisation: lifestyle modifications such as eating small frequent meals, proton pump inhibition and a lowfat diet are only adjuncts, and medical therapy such as long-term anticoagulation is reserved only for patients who cannot undergo revascularisation. The goals of treatment are symptom relief, weight restoration and the prevention of progression to acute mesenteric ischaemia and intestinal infarction [11]. A recent systemic review of 12 studies by Saedon et al. [12] found that endovascular and open surgical revascularisation techniques were associated with statistically similar rates of peri-operative mortality (odds ratio $0.78,95 \% \mathrm{CI} 0.40-1.50$, $\mathrm{p}=0.45$ ). Though associated with longer hospital stays, surgical revascularisation was associated with better long-term patency in 8 studies where this was reported, with a statistically significant odds ratio of 3.57 compared to endovascular revascularisation (95\% CI 1.836.97, $\mathrm{p}=0.0002$ ).

However, in this case it was felt that as the vascular stenosis involved only a short ostial segment, endovascular therapy was the best first-line option especially in a malnourished patient in whom wound healing after open procedures would be theoretically impaired. Reported technical success rates of endovascular treatment are high at $85-100 \%$ with shortterm symptomatic relief in $80-95 \%$ of patients [13]. Though in-stent restenosis may be a future problem in up to $60 \%$ of cases over 2 years, several single-centre series describe favourable outcomes when symptomatic restenosis is treated with further endovascular in- 
Yeoh: A Delayed Diagnosis of Chronic Mesenteric Ischaemia: The Role of Clinicians Cognitive Errors

terventions. Peck et al. [14] reported 13 of 49 (28.6\%) patients undergoing endovascular intervention undergoing repeat endovascular procedures at a median time of 15.5 months. Of these 13 patients, 9 remained completely revascularised and asymptomatic at 3 years after initial intervention. The remaining 4 underwent successful subsequent surgical revascularisation, 3 of whom remained completely revascularised and asymptomatic at 3 years after initial intervention ( 1 died of an unrelated cause). Even if surgical intervention were needed for restenosis, this is theoretically associated with a lower risk of peri-procedural complications compared to first-line surgical therapy as the patient will have undergone an intervening period of nourishment and reconditioning.

The choice to combine balloon angioplasty and stenting for our patient is supported by a systemic review of 328 patients undergoing endovascular intervention in 16 studies [15] suggesting that, while not reaching statistical significance, this approach trends toward higher initial technical success compared to angioplasty alone (92 vs $83 \%, \mathrm{p}=0.09$ ) but decreased restenosis rates compared to stenting alone ( $26 \mathrm{vs.} 35 \%, \mathrm{p}=0.10$ ).

This case highlights the importance of recognising atypical presentations of gastrointestinal conditions as well as considering causes for diarrhoea outside the gut lumen. Importantly, it serves as an illustration of multiple cognitive errors to which clinicians may be susceptible in practice. Awareness of the possibility of such errors allows clinicians to reflect critically on their diagnostic reasoning in difficult cases and reduce diagnostic delay.

\section{Statement of Ethics}

The authors have no ethical conflicts to declare.

\section{Disclosure Statement}

The authors have no conflicts of interest to declare.

\section{References}

1 Thomas JH, Blake K, Pierce GE, Hermreck AS, Seigel E: The clinical course of asymptomatic mesenteric arterial stenosis. J Vasc Surg 1998;27:840-844.

- Kolkman JJ, Bargeman M, Huisman AB, Geelkerken RH: Diagnosis and management of splanchnic ischemia. World J Gastroenterol 2008;14:7309-7320.

-3 Chang RW, Chang JB, Longo WE: Update in management of mesenteric ischemia: World J Gastroenterol 2006;12:3243-3247.

4 Barret M, Martineau C, Rahmi G, Pellerin O, Sapoval M, Alsac JM, Fabiani JN, Malamut G, Samaha E, Cellier C: Chronic mesenteric ischaemia: a rare cause of chronic abdominal pain. Am J Med 2015;128:1363.e1-e8.

5 Schmidt HG, Rikers RM: How expertise develops in medicine: knowledge encapsulation and illness script formation. Med Educ 2007;41:1133-1139.

6 Alibrahim B, Aljasser MI, Salh B: Fecal calprotectin use in inflammatory bowel disease and beyond: a mini-review. Can J Gastroenterol Hepatol 2015;29:157-163.

7 Mamede S, van Gog T, van den Berge K, Rikers RM, van Saase JL, van Guldener C, Schmidt HG: Effect of availability bias and reflective reasoning on diagnostic accuracy among internal medicine residents. JAMA 2010;304:1198-1203.

-8 Croskerry P: Achieving quality in clinical decision making: cognitive strategies and detection of bias. Acad Emerg Med 2002;9:1184-1204.

-9 Artino AR Jr, Durning SJ, Waechter DM, Leary KL, Gilliland WR: Broadening our understanding of clinical quality: from attribution error to situated cognition. Clin Pharmacol Ther 2012;91:167-169. 


\section{Case Reports in Gastroenterology}

\begin{tabular}{l|l}
\hline Case Rep Gastroenterol 2016;10:68-74 \\
\hline 10.1159/000444276 & $\begin{array}{l}\text { @ 2016 The Author(s). Published by S. Karger AG, Basel } \\
\text { www.karger.com/crg }\end{array}$ \\
\hline
\end{tabular}

Yeoh: A Delayed Diagnosis of Chronic Mesenteric Ischaemia: The Role of Clinicians' Cognitive Errors

10 Vázquez-Costa M, Costa-Alcaraz AM: Premature diagnostic closure: an avoidable type of error. Rev Clin Esp (Barc) 2013;213:158-162.

11 Hohenwalter EJ: Chronic mesenteric ischemia: diagnosis and treatment. Semin Intervent Radiol 2009;26:345-51.

$\$ 12$ Saedon M, Saratzis A, Karim A, Goodyear S: Endovascular versus surgical revascularization for the management of chronic mesenteric ischemia. Vasc Endovascular Surg 2015;49:37-44.

13 Loffroy R, Steinmetz E, Guiu B, Molin V, Kretz B, Gagnaire A, Bouchot O, Cercueil JP, Brenot R, Krausé D: Role for endovascular therapy in chronic mesenteric ischemia. Can J Gastroenterol 2009;23:365-373.

14 Peck MA, Conrad MF, Kwolek CJ, LaMuraglia GM, Paruchuri V, Cambria RP: Intermediate-term outcomes of endovascular treatment for symptomatic chronic mesenteric ischemia. J Vasc Surg 2010;51:140-147.e1-e2.

15 Kougias P, El Sayed HF, Zhou W, Lin PH: Management of chronic mesenteric ischemia: the role of endovascular therapy. J Endovasc Ther 2007;14:395-405.

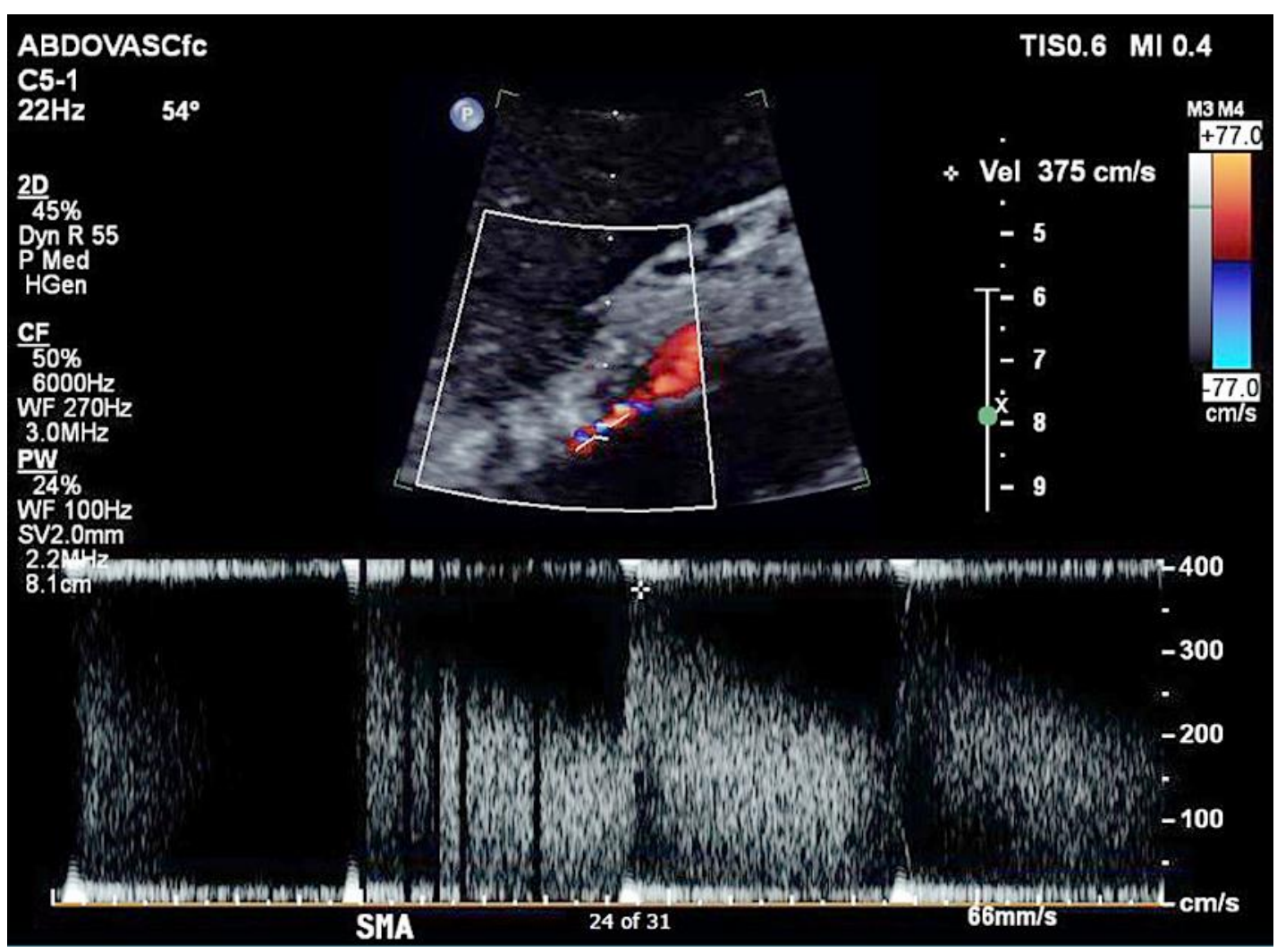

Fig. 1. Doppler ultrasound of the superior mesenteric artery showing elevated peak systolic velocity of 375 $\mathrm{cm} / \mathrm{s}$ corresponding to $>70 \%$ stenosis. 
\begin{tabular}{ll|l} 
Case Reports in & $\begin{array}{l}\text { Case Rep Gastroenterol 2016;10:68-74 } \\
\text { Gastroenterology }\end{array}$ & $\begin{array}{l}\text { O 2016 The Author(s). Published by S. Karger AG, Basel } \\
\text { www.karger.com/crg }\end{array}$ \\
\cline { 2 - 3 } & Yeoh: A Delayed Diagnosis of Chronic Mesenteric Ischaemia: The Role of Clinicians'
\end{tabular}

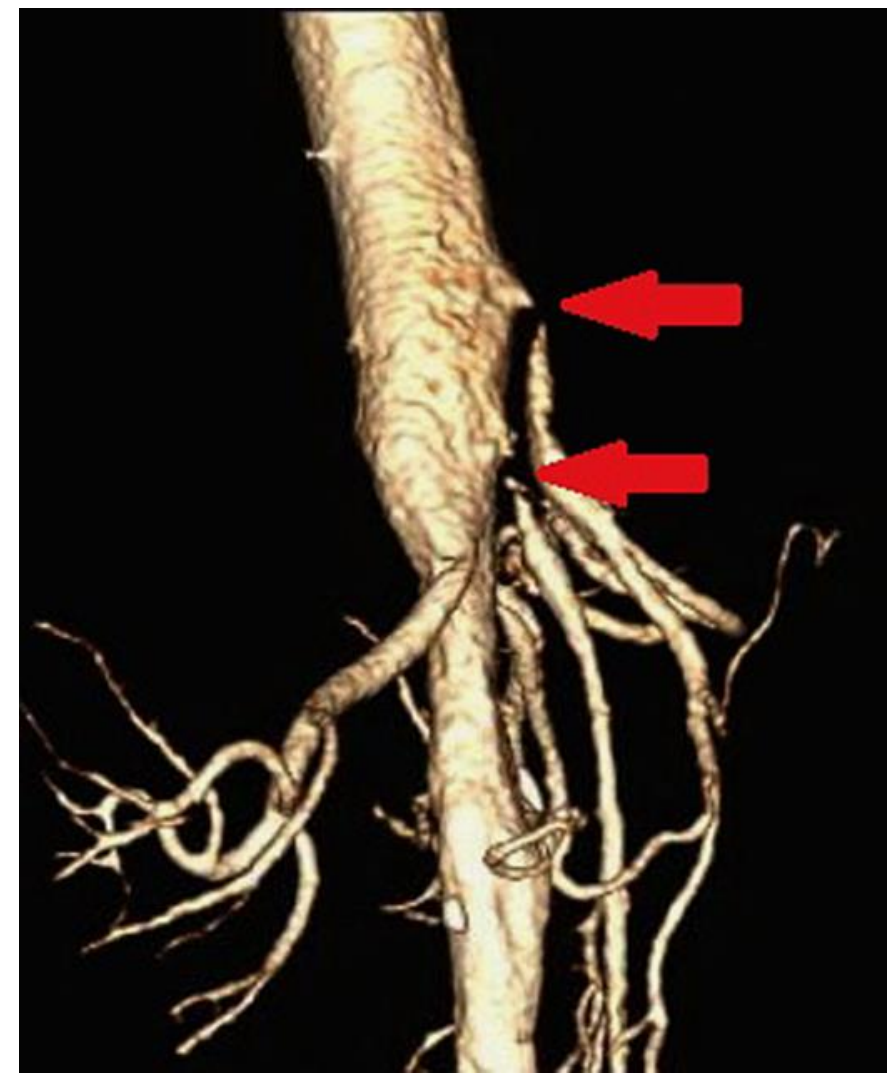

Fig. 2. Three-dimensional CT reconstruction of the aorta and mesenteric vessels demonstrating $90 \%$ ostial stenosis in the coeliac artery (top arrow) and superior mesenteric artery (bottom arrow).

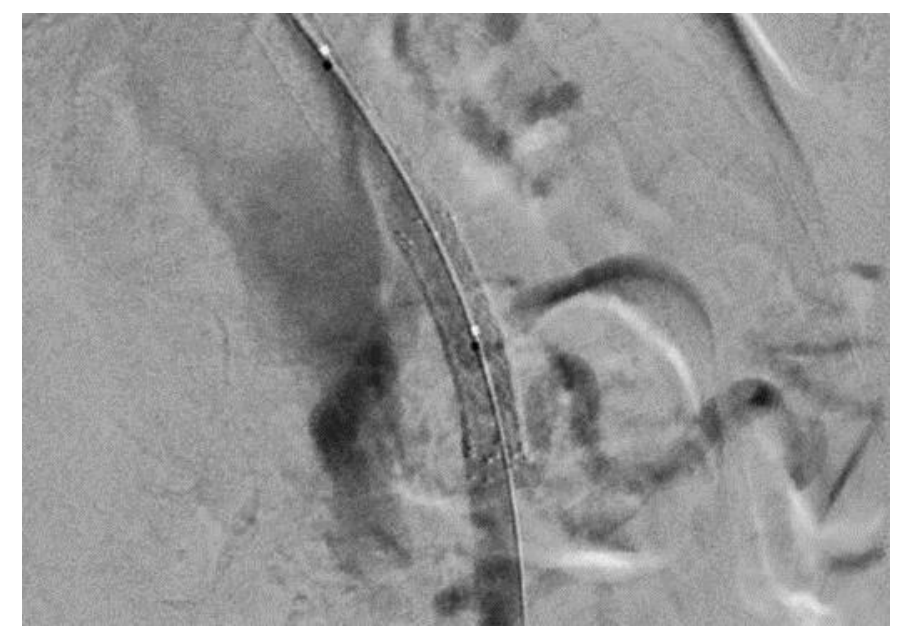

Fig. 3. Fluoroscopic image of the superior mesenteric artery with guidewire and self-expanding stent in situ. 